\title{
Thyroid antoantibodies and the response to thyrotropin releasing hormone in patients with subclinical hypothyroidism
}

\author{
O A Mojiminiyi, V Rege, J Bolodeoku, A H Wilcox, J L Barron
}

\begin{abstract}
Aim-To evaluate the clinical usefulness of the thyrotropin releasing hormone (TRH) test and estimation of thyroid autoantibody concentrations in patients with borderline raised thyroid stimulating hormone (TSH).

Methods-The records of 34 consecutive patients with persistent borderline increased TSH (4.4-9.9 mU/1) referred to the Medical Investigation Unit were reviewed. The response of patients with thyroid autoantibodies to the TRH test was compared with that of patients with a negative antibody screen.

Results-Eleven (44\%) of 25 patients with positive anti-thyroid microsomal and/or thyroglobulin antibody tests and three (33\%) of nine patients with a negative antibody screen had hypothyroid responses to TRH. Neither age nor sex affected the response to TRH. Basal TSH alone was poorly correlated with these indices. Twelve (35\%) patients who had elevated basal TSH had a normal response to the TRH test.

Conclusion-Patients with positive or negative thyroid autoantibodies and an exaggerated response to the TRH test should be regarded as hypothyroid and treated with thyroxine. Patients with positive thyroid autoantibodies and normal TSH response may subsequently develop hypothyroidism and should be given long term follow up.

(F Clin Pathol 1995;48:463-465)
\end{abstract}

Keywords: Hypothyroidism, thyroid stimulating hormone, thyrotropin releasing hormone.

Subclinical hypothyroidism, a condition in which clinically euthyroid patients have borderline raised serum thyroid stimulating hormone (TSH 4.3-9.9 mU/l) and a normal thyroxine concentration on at least two occasions, often causes diagnostic and management problems. Although the condition has been recognised for over 25 years, ${ }^{1}$ little is known about its course and there is no consensus on the management of these patients. A borderline raised TSH concentration and the presence of thyroid autoantibodies are biochemical features used to identify people at risk of progression to overt hypothyroidism. ${ }^{2}$ It has been suggested that those with raised TSH concentrations and positive thyroid microsomal antibodies may be treated with thy- roxine whilst for those without antibodies the raised TSH concentrations should be disregarded. ${ }^{3}$

Performance of the thyrotropin releasing hormone (TRH) test is often used as an additional test in these patients. ${ }^{45}$ The availability of sensitive and ultrasensitive assays for $\mathrm{TSH}$ and much improved assays for free thyroid hormones have limited the indications for the performance of the TRH test, especially in patients with suspected thyroid disease. This study reviews the contribution of the TRH test and thyroid antibodies to the management of patients with suspected subclinical hypothyroidism referred to a Medical Investigation Unit over an 18 month period.

\section{Methods}

The records of 34 ( 30 women and four men) consecutive patients referred over an 18 month period were reviewed. The patients were aged between 23 and 80 years $(58 \pm 13.9$ SEM). All of the patients had borderline raised TSH concentrations $(4 \cdot 4-9 \cdot 9 \mathrm{mU} / \mathrm{l})$ on at least two occasions three to six months apart.

Total thyroxine was measured by radioimmunoassay (Immunodiagnostic Systems Ltd, Tyne and Wear, UK); TSH was measured using a coated tube immunoradiometric assay (Immunodiagnostic Systems Ltd). Interassay precision (coefficient of variation) for the TSH assay was $7 \%$ at $3.9 \mathrm{mU} / 1$ and $6 \%$ at $26.5 \mathrm{mU} / 1$. Thyroglobulin antibodies and thyroid microsomal antibodies were assessed by passive haemagglutination using Serodia kits (Fujirebio Inc., Tokyo, Japan). The TRH test was performed by intravenous administration of $200 \mu \mathrm{g}$ TRH and blood samples were collected at zero, 20 and 60 minutes.

The indices used in the diagnosis of hypothyroidism were increment of TSH $>30 \mathrm{mU} / 1$, 20 minute $\mathrm{TSH}>35 \mathrm{mU} / 1$ or a proportional rise (20 minute $\mathrm{TSH} / \mathrm{basal} \mathrm{TSH}>6$ ).

\section{Results}

Administration of TRH was well tolerated by all of the patients and no side effects were reported. The mean total thyroxine concentration in the patients was $92 \pm 2 \cdot 7$ (SEM) $\mathrm{nmol} / \mathrm{l}$ (range $60-112 \mathrm{nmol} / \mathrm{l}$ ). Using linear regression analysis, there was good correlation between the indices used for the diagnosis of hypothyroidism $(r \geq 0.76$; $\mathrm{p} \leq 0.001)$. Basal TSH alone was poorly correlated with these indices $(r \leq 0 \cdot 145 ; p \leq 0.46)$ 

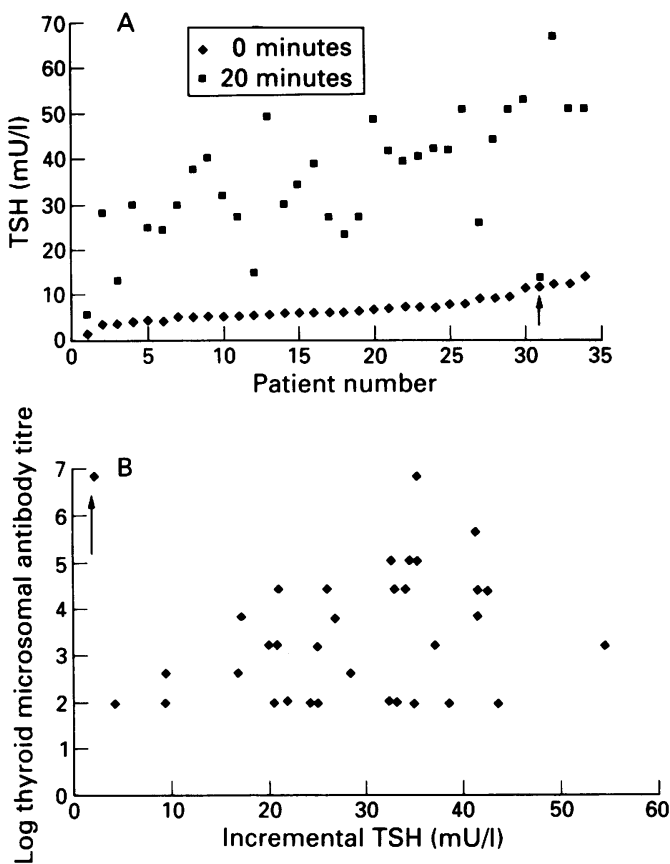

A: TSH response to administration of TRH in patients with borderline raised TSH concentrations. The arrow indicates a patient with a subnormal response to TRH who was later diagnosed as having a pitiutary tumour. B: Correlation between the TSH response to TRH and thymid microsomal antibody titre in patients with persistent borderline raised TSH concentrations. Normal incremental TSH is 5-25 mU/l; normal log microsomal antibody titre <2; arrow as in $A$.

and $12(35 \%)$ patients who had a borderline raised basal TSH concentration had a normal response to the TRH test. The TSH response to TRH administration is shown in the figure (panel A). There were no age or sex related differences in incremental TSH or the proportional rise in TSH concentrations in the patients studied. One patient who had a subnormal response (incremental TSH $=2 \cdot 1 \mathrm{mU} / \mathrm{l}$ ) was later diagnosed as having a pituitary tumour causing hypopituitarism (panel A).

Of the 24 patients with positive thyroid microsomal and/or thyroglobulin antibody tests, $11(44 \%)$ had hypothyroid responses. Nine patients were negative for thyroid autoantibodies and three $(33 \%)$ of these had hypothyroid responses. The correlation between incremental TSH and logarithm of the thyroid microsomal antibody titre is shown in the figure (panel B). The difference between the mean increment in TSH between the two groups was $2.93 \mathrm{mU} / 1$ (95\% confidence interval -7.63 to 13.5) and this was not statistically significant $(\mathrm{p}=0.69)$, indicating that thyroid autoantibodies cannot predict $\mathrm{TSH}$ response to TRH stimulation in these patients.

\section{Discussion}

The incidence of the detection of patients with borderline raised TSH concentrations has been increasing mainly because of increased screening of elderly people. Serum TSH concentration has often been proposed as the test of choice for screening for thyroid disease in the elderly ${ }^{36}$ but the serum TSH concentration is affected by a number of factors including recent acute illness when a borderline TSH concentration could be transient. ${ }^{7}$ For these reasons, the criteria used for selecting patients for this study included persistent borderline elevation of TSH concentrations on two occasions at least three to six months apart. A prospective study to evaluate the course of borderline raised $\mathrm{TSH}$ concentrations identified two groups of patients: (1) those with true subclinical hypothyroidism who may be predicted by various aetiological factors; and (2) patients with reset thyrostat who have persistently elevated TSH concentrations without progression to hypothyroidism. ${ }^{5}$

Development of overt hypothyroidism is a graded phenomenon and a borderline raised TSH represents progression to gland failure in many patients. ${ }^{68}$ Some studies have shown that patients with elevated serum TSH concentrations and thyroid autoantibodies are more likely to develop hypothyroidism ${ }^{29}$ and may be treated to avoid progression to hypothyroidism. ${ }^{26}$ This study confirms previous reports which showed that thyroid autoantibodies do not necessarily indicate the presence of thyroid dysfunction. ${ }^{1011}$ Raised basal TSH concentrations and the presence of thyroid autoantibodies did not correlate with the response to the TRH test, indicating that neither the basal TSH concentration nor thyroid autoantibodies could be used to predict the result of the TRH test. It is known that the response to the TRH test may be blunted as a result of reduced thyrotroph reserve, ${ }^{12}$ but thyroid function has been shown to be well preserved until the eighth decade in healthy subjects. ${ }^{13}$ It is unlikely that the results in this study could have been affected by the age of the patients which spanned seven decades.

There is a debate whether borderline raised TSH concentrations or subclinical hypothyroidism is a biochemical syndrome but the condition poses an increased risk for development of cardiovascular disease due to lipid abnormalities ${ }^{1}$ and treatment with L-thyroxine has been shown to be beneficial. ${ }^{14}$ The decision to treat and identification of individuals with borderline raised TSH concentrations who would benefit from treatment pose practical problems. Treatment of all patients would result in unnecessary treatment of a large number of asymptomatic patients and unknown cost/benefit implications. ${ }^{2}$ One study which showed that approximately one woman in four with the condition would benefit from treatment ${ }^{15}$ concluded that those who would benefit may be identified by low serum tri-iodothyronine or free tri-iodothyronine concentrations, both of which are poor indicators of the degree of hypothyroidism. Determining whether therapy is appropriate requires biochemical as well as clinical evaluation. The results of the present study indicate that patients with subclinical hypothyroidism and exaggerated response to TRH should be treated with thyroxine whether or not their thyroid autoantibody screen is positive. Although patients with positive thyroid autoantibodies and normal TSH response can subsequently develop hypothyroidism, there is considerable debate on their immediate management. This 
is further complicated by the reported prevalence of thyroid autoantibodies in an apparently normal and healthy elderly population. ${ }^{11}$ For this group of patients, a prospective long term follow up study will be useful for the determination of treatment criteria. This should include regular assessment of thyroid, cardiovascular and lipid status.

1 Bastenie PA, Vanhaelst L, Neve P. Coronary artery disease in hypothyroidism: observations in preclinical myxoedema. hancet 1967;ii:1221-2.

2 Tunbridge W, Brewis M, French J, Appleton D, Bird T, Clark F, et al. Natural history of autoimmune thyroiditis. Clark F, et al. Natural hist

3 Rae P, Farrar J, Beckett G, Toft A. Assessment of thyroid status in elderly people. BMF 1993;307:177-80.

4 Brown CA, Hennessy JV. Clinical significance of mildly elevated thyrotropin levels with normal thyroxine levels. South Med $\mathcal{F}$ 1989;82:681-5.

5 Kabadi MU. "Subclinical hypothyroidism": natural course of the syndrome during a prolonged follow-up study. Arch Intern Med 1993;153:957-61.

6 Tibaldi J, Barzel US. Thyroxine supplementation: Method for the prevention of clinical hypothyroidism. $\mathrm{Am} \mathcal{f} \mathrm{Med}$ 1985;79:241-4.
7 Hamblin PS, Dyer SA, Mohr VS, Le Grand BA, Lim CF, Tuxen DV, et al. Relationship between thyrotropin and thyroxine changes during recovery from severe hypothyroxinaemia of critical illness. $\mathcal{F}$ Clin Endocrinol Metab 1986;62:717-22.

8 Evered DC, Ormston BJ, Smith PA, Hall R, Bird T. Grades of hypothyroidism. BMF 1973;i:657-62.

9 Gordin A, Lamberg BA. Natural course of symptomless autoimmune thyroiditis. Lancet 1975;ii:1234-8.

10 Robuschi G, Safran M, Braverman L, Gnudi A, Roti E. Hypothyroidism in the elderly. Endocr Rev 1987;8:142-53.

11 Roti E, Gardini E, Minelli R, Bianconi L, Braverman E. Prevalence of anti-thyroid peroxidase antibodies in serum in the elderly: comparison with other tests of anti-thyroid antibodies. Clin Chem 1992;38:88-92.

12 Finucane P, Rudra R, Hsu R, Newcombe R, Pathy MS, Scanlon MF, et al. Thyrotropin response to thyrotropinreleasing hormone in elderly patients with and without acute illness. Age Ageing 1991;20:85-9.

13 Mariotti S, Barbesina G, Caturegli P, Bartalena L, Sansoni $\mathrm{P}$, Fagoni $\mathrm{F}$, et al. Complex alteration of thyroid function in healthy centenarians. $\mathcal{F}$ Clin Endocrinol Metab 1993;77: in healthy

14 Caron P, Calazel C, Para HJ, Hoff M, Louvet JP. Decreased HDL cholesterol in subclinical hypothyroidism: the effect of L-thyroxine therapy. Clin Endocrinol 1990;33:519-23.

15 Nyström E, Caidahl K, Fager G, Wikkelso C, Lundberg PA, Lindstedt G. A double-blind cross-over 12-month study of L-thyroxine treatment of women with "subclinical” hypothyroidism. Clin Endocrinol 1988;29:221-3. 